\title{
Association between grade of hypertension and sensorineural hearing loss
}

\author{
Naveen Kumar Korivipati ${ }^{1}$, Pavan Kumar Mangalam²*, Saadia Butool ${ }^{3}$, Ayesha Ather Hussain ${ }^{4}$, \\ Zaiba kousar ${ }^{5}$, Mohd Inamul Haq ${ }^{6}$
}

${ }^{1}$ Professor, ${ }^{2}$ Assistant Professor, ${ }^{3}$ Senior Resident, 4,5,6PG, Department of Otolaryngology, Shadan Institute of Medical Science, Shadan Hospital, Himayathsagar Road, Hyderabad-08, Telangana State, INDIA.

Email: aisha7311@gmail.com

Abstract Background: Hearing loss is the most frequent sensory deficit in the human population. The prevalence of hypertension in developing countries is increasing rapidly. Hypertension causes increased blood viscosity, which reduce capillary blood flow and causes tissue hypoxia, thus causing hearing loss in patients. Aim: To evaluate the association between grade of hypertension and sensorineural hearing loss. Material and Methods: A total of 102 hypertensive patients, aged 45 to 65 years on treatment presenting with sensorineural hearing loss. Blood pressure was measured on two occasions, 6 hours apart, in the sitting position using a standard mercury sphygmomanometer with an adult cuff size. Hearing thresholds were then measured in both ears using pure tone audiometry. Results: Patients were divided into five subgroups based on type of sensorineural hearing loss. Percentage of patients with mild degree of sensorineural hearing loss was 15 , that with moderate degree was 22 , that with moderately severe was 12 , with severe was 15 and that with profound was 6. The grade of hypertension also showed a significant influence on sensorineural hearing loss. Conclusion: This cohort study confirms that there is a possible association between hypertension and sensorineural hearing loss as demonstrated by higher prevalence and raised hearing threshold levels by pure tone audiometry in all frequencies in hypertensive patients.

Key Words: Hypertension, sensorineural hearing loss, pure tone audiometry, severity.

*Address for Correspondence:

Dr. Pavan Kumar Mangalam, H. No 12-6-36/4/9, Flat no. 104, MJR Solitaire, Moosapet 500018

Email: pavan.mangalam@gmail.com

Received Date: 08/10/2019 Revised Date: 20/11/2019 Accepted Date: 11/12/2019

DOI: https://doi.org/10.26611/10161511

This work is licensed under a Creative Commons Attribution-NonCommercial 4.0 International License. $(\mathrm{cc})$ BY-NC

\begin{tabular}{|l|l|}
\hline \multicolumn{2}{|c|}{ Access this article online } \\
\hline Quick Response Code: & Website: \\
www.medpulse.in \\
\end{tabular}

\section{INTRODUCTION}

Hearing loss is any degree of impairment of the ability to comprehend sound. It is partial or total inability to hear sound in either one or both ears. Hearing loss is the most frequent sensory deficit in the human population with $\mathrm{WHO},{ }^{1}$ estimating 360 million people in the world to have disabling hearing loss of which 328 million (91\%) are adults. The hearing loss (HL) is a factor that irrespectively of the degree of commitment affects the quality of life and when acquired in adults, it appears gradually and may make the oral language receiving difficulty. Fifty percent of the causes are avoidable by prevention or early diagnosis and treatment. Hypertension is one of the causative factors. The prevalence of hypertension in developing countries is increasing rapidly despite it being a preventable disease in a significant number of cases. ${ }^{2}$ High pressure in the vascular system may cause inner ear hemorrhage, which is supplied by the anterior inferior cerebellar artery, which supports the inner ear artery and is divided into cochlear artery and anterior vestibular artery, which may cause progressive or sudden hearing loss. ${ }^{3}$ This circulatory system pathology may directly affect hearing in a number of ways. One of the vascular physiopathological mechanisms described is the increase in blood viscosity, which reduce capillary blood flow and ends up reducing oxygen transport, causing tissue hypoxia, thus causing hearing complaints and hearing loss in patients. Moreover, arterial 
hypertension may cause ionic changes in cell potentials, thus causing hearing loss. The present case control study was conducted to evaluate the association between grade of hypertension and sensorineural hearing loss.

\section{MATERIAL AND METHODS}

This case control study was conducted over a period of two years in the Department of ENT and outpatient department of General medicine at a tertiary care research center. The study sample comprised of randomly selected hypertensive patients, aged 45 to 65 years on treatment at medicine OPD presenting with sensorineural hearing loss (SNHL).

\section{Sample Size}

A total of 102 patients who satisfied the selection criteria and consented were included in the study.

\section{Inclusion criteria}

- Both sexes

- Diagnosed cases of Hypertension as approved under revised diagnostic criteria of HTN guidelines. (British antihypertensive society)

- Patients on treatment of HTN but were still having fluctuating blood pressure

\section{Exclusion criteria}

- Non hypertensive patients

- Age $<45$ years and $>65$ years

- Family history of deafness

- History of ear problems (discharge)

- History of head injury or meningitis

\section{Methodology}

A detailed information about the auditory and vestibular disorders symptomatology, presence of tinnitus, as well as exposure to hearing harmful agents, such as occupational or leisure noise, ototoxic drugs and general health conditions was noted. Blood pressure was measured on two occasions, 6 hours apart, in the sitting position using a standard mercury sphygmomanometer with an adult cuff size. Patients with arterial blood pressure $\geq 140 / 90 \mathrm{~mm}$ of $\mathrm{Hg}$ in different measures, were considered positive for high blood pressure. Both ears of the participants were examined. Any obstructing wax observed during otoscopy was removed, after softening in outpatient department. Hearing thresholds were then measured in both ears using the Modified HughsonWestlake method4 at 250, 500, 1000, 2000, 4000, 6000, and $8000 \mathrm{~Hz}$ for air conduction and 500, 1000, 2000, and
$4000 \mathrm{~Hz}$ for bone conduction using a Diagnostic Audiometer in a sound isolated room, which satisfied the criteria of ISO 8253-1. Average of audiometric hearing thresholds at 500,1000, 2000, and $4000 \mathrm{~Hz}$ for both air and bone conduction was determined, which was taken to be the pure tone average for both air and bone conduction. This was categorized in accordance to the WHO grades of hearing impairment as follows: normal hearing $(<25 \mathrm{~dB})$, mild hearing loss $(26-40 \mathrm{~dB})$, moderate hearing loss (41-60 dB), severe hearing loss (61-80 dB), and profound hearing loss $(\geq 81 \mathrm{~dB}) .5$ The hearing thresholds for the better ear was used for further analysis in both patient and control groups.

Ethical approval taken from Institutional ethical committee.

\section{RESULTS}

Total number of patients studied were 102 with age ranging from 45-65 years. They were divided into 4 groups. In group 1 (45-50 years) total patients were 41 out of 102, in group 2 (51-55years) total patients were 23 out of 102, in group 3 (56-60years) total patients were 12 out of 102, finally in group 4 (61-65years) total patients were 26 out of 102 . Again patients were divided according to their gender into two sub-groups. Males were 49 out of 102 and females were 53 out of 102 . Patients were graded into three grades according to British Hypertension society, grade 1 includes 26, grade 2 includes 52 and grade 3 includes 24 .

Table 1: Grades of Hypertension

\begin{tabular}{ccc}
\hline Grade of HTN & Total & Percentage \\
\hline Grade 1 & 26 & $25.49 \%$ \\
Grade 2 & 52 & $50.98 \%$ \\
Grade 3 & 24 & $23.52 \%$ \\
\hline Total & 102 & $100 \%$ \\
\hline
\end{tabular}

Patients are divided into three groups based on duration of sensorineural hearing loss post hypertension subgroup A includes 13 cases, subgroup B includes 34 cases, subgroup $\mathrm{C}$ includes $23 \%$. Patients are divided into five subgroups based on type of sensorineural hearing loss. Percentage of patients with mild degree of sensorineural hearing loss was 15 , that with moderate degree was 22 , that with moderately severe was 12 , with severe was 15 and that with profound was 6 .

\begin{tabular}{ccc}
\multicolumn{3}{c}{ Table 2: Type of sensorineural hearing loss } \\
\hline Type of SNHL & Total & Percentage \\
\hline Mild & 15 & $21.42 \%$ \\
Moderate & 22 & $31.42 \%$ \\
Moderately severe & 12 & $17.14 \%$ \\
Severe & 15 & $21.42 \%$ \\
Profound & 6 & $8.57 \%$ \\
\hline Total & 70 & $100 \%$ \\
\hline
\end{tabular}


Table 3: Association of grade of hypertension with type of SN hearing loss

\begin{tabular}{|c|c|c|c|c|c|c|c|c|c|}
\hline & & & \multicolumn{6}{|c|}{ Type of SN hearing loss } & \multirow[t]{2}{*}{ Total } \\
\hline & & & Mild & Mod & Mod-sev & Norm & Prof & Sev & \\
\hline \multirow{6}{*}{ Grade of HTN } & & Count & 11 & 3 & 0 & 4 & 0 & 8 & 26 \\
\hline & Mild & $\%$ within Grade of HTN & $42.3 \%$ & $11.5 \%$ & $0.0 \%$ & $15.4 \%$ & $0.0 \%$ & $30.8 \%$ & $100 \%$ \\
\hline & \multirow{2}{*}{ Moderate } & Count & 3 & 13 & 12 & 17 & 2 & 5 & 52 \\
\hline & & $\%$ within Grade of HTN & $5.8 \%$ & $25.0 \%$ & $23.1 \%$ & $32.7 \%$ & $3.8 \%$ & $9.6 \%$ & $100 \%$ \\
\hline & \multirow{2}{*}{ Severe } & Count & 1 & 6 & 0 & 11 & 4 & 2 & 24 \\
\hline & & $\%$ within Grade of HTN & $4.2 \%$ & $25.0 \%$ & $0.0 \%$ & $45.8 \%$ & $16.7 \%$ & $8.3 \%$ & $100 \%$ \\
\hline \multirow{2}{*}{\multicolumn{2}{|c|}{ Total }} & Count & 15 & 22 & 12 & 32 & 6 & 15 & 102 \\
\hline & & $\%$ within Grade of HTN & $14.7 \%$ & $21.6 \%$ & $11.8 \%$ & $31.4 \%$ & $5.9 \%$ & $14.7 \%$ & $100.0 \%$ \\
\hline
\end{tabular}

$\mathrm{SN}=$ sensorineural; Mod= Moderate; Sev= severe; Norm=Normal; Prof=profound

\begin{tabular}{cccc}
\hline \multicolumn{4}{c}{ Chi-Square Tests } \\
\hline Value & df & Asymp. Sig. (2-sided) \\
Pearson Chi-Square & $47.802 \#$ & 10 & 0.000 \\
Likelihood Ratio & 49.102 & 10 & 0.000 \\
N of Valid Cases & 102 & & \\
\hline \# 9 cells (50\%) have expected count less than 5. The minimum \\
expected count is 1.41.
\end{tabular}

The grade of hypertension showed a significant influence on sensorineural hearing loss $(\mathrm{P}-\mathrm{value}=0.000)$.

\section{DISCUSSION}

Hearing loss which has multifactorial causation is currently a public health concern. Several studies in developed countries have been conducted to find out whether hypertension is one of the risk factors for hearing loss. The results have been contradictory with some showing positive correlation ${ }^{6-8}$ while others have shown no relation between hearing loss and hypertension ${ }^{9,10}$ with that in mind this study was conducted to ascertain whether hypertension is a risk factor for hearing loss in our setup where such data is not available. As to the methodological characteristics of this study, the case is taken in outline with the age factor, focusing on the age range of middle aged individuals, between 45 and 65 years, all being hypertensive patients. The strict exclusion criteria, eliminating individuals without hypertension and other specific conditions capable of producing hearing alterations. With aging, there is a higher number of chronic diseases. In this study we observed that although the sample individuals were between 45 and 65 years, higher age range proved not to be associated with hearing loss. Some studies justify that sensorineural hearing loss that happens with aging is related to microcirculatory insufficiency that occurs due to vascular occlusion caused by emboli, hemorrhage or vasospasm and these happen because of a syndrome of hyperviscosity or microangiopathy caused by diabetes or hypertension and the latter could, through histopathological mechanisms cause sensorineural hearing loss. As to gender, there was a difference in men to women ratio due to the fact that we did not pair the sample, cases were taken at random, during regular medical visits. Many studies have shown relation between gender and hearing loss in relation to age. ${ }^{6}$ In present study carried out in individuals with ages varying between 45 and 65 years, gender proved insignificantly associated with hearing loss. However, the studies by Chen et $a l^{11}$ with $100 \%$ and Agarwal et $a l^{7}$ with $65.8 \%$ had higher numbers for male subjects. This may account for some differences in the results as males are more predisposed to hearing loss as shown by Pearson et al. ${ }^{12}$ Eleven out of one hundred and two hypertensive patients with hearing loss $(10.78 \%)$ had tinnitus, while seventeen out of one hundred and two hypertensive patients with hearing loss (17\%) had vertigo. Hypertension as a risk factor for tinnitus has been demonstrated in other studies. Hypertension has been associated with hearing loss ${ }^{6-8}$ which is thought to occur due to microcirculatory insufficiency. In our study patients with hypertension were at higher risk of hearing loss and this is statistically significant at a P-value of 0.01 . Our findings are similar to other studies ${ }^{6-8}$ which have shown a link between high blood pressure and sensorineural hearing loss. The grade of hypertension also showed a significant influence on sensorineural hearing loss $(\mathrm{P}$-value $=0.000)$.

\section{CONCLUSION}

This cohort study confirms that there is a possible association between hypertension and sensorineural hearing loss as demonstrated by higher prevalence and raised hearing threshold levels by PTA in all frequencies in hypertensive patients. The increase in hearing thresholds was most marked among those with grade 3 hypertension and among hypertensive patients, moderate degree of sensorineural hearing loss was most marked as per our study. 


\section{REFERENCES}

1. World Health Organization. WHO global estimates on prevalence of hearing loss based on 42 population based studies (internet) 2012 [cited Oct 2019 available from httpp://www.who.int/pbd/deafness/estimates.

2. Joshi MD, Ayah R, Naju EK, et al. Prevalence of hypertension and associated cardiovascular risk factors in urban slum, Kenya. Population based survey. BMC public health 2014;14:1177.

3. Kim HA, Lee H. Recent Advances in Understanding Audiovestibular Loss of a Vascular Cause. J Stroke. 2017;19(1):61-66.

4. Arlinger S. Psychoacoustic audiometry. In: Gleeson M, Browning GG, Burton MJ, Clarke R, Hibbert J, Jones NS et al. editors. Scott - Brown's otolaryngology, head and neck surgery, 7th ed. London: Hodder Arnold 2008. pp. 3260-3275.

5. World Health Organization. Prevention of blindness and deafness: grades of hearing impairment. Geneva: World Health Organization 2014.

6. de Moraes Marchiori LL, de Almeida Rego Filho E, Matsuo T. Hypertension as a factor associated with hearing loss. Rev Bras Otorhinolaryngol 2006;72(4).
7. Agarwal S, Mishra A, Jagade M, et al. Effects of hypertension on hearing. Ind J Otolaryngol Head and neck surgery. 2013;65(Suppl 3):614-618

8. Mondelli MFCG, Lopes AC. Relation between Arterial Hypertension and hearing loss. Int Arch Otorhinolaryngol 2009;13(1):63-68.

9. Altuntas E E, Yenicesu AG, Mutlu AE, et al. An evaluation of the effects of hypertension during pregnancy on postpartum hearing as measured by transient evoked otoacoustic emissions. Acta Otorhinolaryngol Ital 2012;32(1):31-36.

10. Hutchinson KM, Alessio H, Baiduc RR. Association between cardiovascular health and hearing functions: Pure tone and distortion product otoaucostic emission measures. Am J Audiol 2010;19:26-35.

11. Chen YL, Ding YP. Relationship between hypertension and hearing disorders in elderly. East AFR Med J 1999;76(6);3:344-347.

12. Pearson JD, Morrell CH, Gordon-Salanta S, et al. Gender differences to longitudinal study of age-associated hearing loss. Int J Pharma Sci Rev Res 2014;29(1);309-313.

\section{Source of Support: None Declared Conflict of Interest: None Declared}

Policy for Articles with Open Access:

Authors who publish with MedPulse International Journal of ENT (Print ISSN: 2579-0854) (Online ISSN: 2636-4727) agree to the following terms: Authors retain copyright and grant the journal right of first publication with the work simultaneously licensed under a Creative Commons Attribution License that allows others to share the work with an acknowledgement of the work's authorship and initial publication in this journal.

Authors are permitted and encouraged to post links to their work online (e.g., in institutional repositories or on their website) prior to and during the submission process, as it can lead to productive exchanges, as well as earlier and greater citation of published work. 